\title{
A Reduction in Consistency Strength for Universal Indestructibility
}

by

\author{
Arthur W. APTER and Grigor SARGSYAN
}

Presented by Stanisław KWAPIEN

Summary. We show how to reduce the assumptions in consistency strength used to prove several theorems on universal indestructibility.

In [3], the first author and Hamkins introduced the concept of universal indestructibility and established several theorems concerning this notion, most prominently the relative consistency of universal indestructibility for both supercompactness and strong compactness. In [1], the first author extended this work and showed the relative consistency of two strongly compact cardinals with universal indestructibility for both supercompactness and strong compactness. All of these results were proven using a high-jump cardinal, a very strong notion reflected by almost hugeness.

The purpose of this paper is to reduce the consistency strength used to prove each of these theorems from a high-jump cardinal to something reflected by this notion which we will call a cardinal Woodin for supercompactness. Specifically, we prove the following two theorems.

THEOREM 1. Universal indestructibility for supercompactness in the presence of a supercompact cardinal is consistent relative to the existence of a cardinal Woodin for supercompactness.

2000 Mathematics Subject Classification: 03E35, 03E55.

Key words and phrases: universal indestructibility, indestructibility, measurable cardinal, Woodin cardinal, strongly compact cardinal, supercompact cardinal, cardinal Woodin for supercompactness, high-jump cardinal, almost huge cardinal.

The first author's research was partially supported by PSC-CUNY Grants and CUNY Collaborative Incentive Grants. 
THEOREM 2. Universal indestructibility for either supercompactness or strong compactness in the presence of two strongly compact cardinals is consistent relative to the existence of a cardinal Woodin for supercompactness.

As we will also indicate without proving explicitly, other theorems from [3] are consistent relative to the existence of a cardinal Woodin for supercompactness.

Before continuing, we take this opportunity to remind readers of some of the relevant definitions. We say that universal indestructibility for supercompactness holds in a model $V$ for ZFC if every $V$-supercompact and partially supercompact (including measurable) cardinal $\delta$ has its degree of supercompactness fully Laver indestructible [7] under $\delta$-directed closed forcing. Analogously, universal indestructibility for strong compactness holds in a model $V$ for ZFC if every $V$-strongly compact and partially strongly compact (including measurable) cardinal $\delta$ has its degree of strong compactness fully indestructible under $\delta$-directed closed forcing. We recall from [3] that the cardinal $\kappa$ is a high-jump cardinal if there is an elementary embedding $j: V \rightarrow M$ having critical point $\kappa$ such that for some $\theta$ we have $M^{\theta} \subseteq M$ and $j(f)(\kappa)<\theta$ for every function $f: \kappa \rightarrow \kappa$. As Lemma 2 of [3] indicates, if $\kappa$ is almost huge, then $\kappa$ is the $\kappa$ th high-jump cardinal.

Our key new concept is that of a cardinal $\kappa$ being Woodin for supercompactness. This will hold if for every $f: \kappa \rightarrow \kappa$ with $f(\alpha)$ a cardinal, there is some $\delta<\kappa$ with $f^{\prime \prime} \delta \subseteq \delta$ and an elementary embedding $j: V \rightarrow M$ having critical point $\delta$ generated by a supercompact ultrafilter having rank below $\kappa$ such that $M^{j(f)(\delta)} \subseteq M$. Our terminology comes from the usual definition of a Woodin cardinal. Since by its definition, a cardinal Woodin for supercompactness is also a Woodin cardinal, it follows that if $\kappa$ is Woodin for supercompactness, then $\kappa$ is both regular and a limit of measurable cardinals (and as Lemma 1.3 will show, much more). In addition, essentially the same proof used with Woodin cardinals shows that the least cardinal Woodin for supercompactness is not weakly compact.

The following lemma is central to establishing our results.

LEMMA 1.1. If $\kappa$ is a high-jump cardinal, then $\kappa$ carries a normal measure concentrating on $A=\{\delta<\kappa \mid \delta$ is Woodin for supercompactness $\}$.

Proof. Our proof is reminiscent of the usual proof that a superstrong cardinal has a normal measure concentrating on Woodin cardinals (see Proposition 26.12 of [6]). Suppose $j: V \rightarrow M$ is an elementary embedding witnessing that $\kappa$ is a high-jump cardinal, with $\theta$ such that $M^{\theta} \subseteq M$ and $j(f)(\kappa)<\theta$ for every $f: \kappa \rightarrow \kappa$. Fix $f: \kappa \rightarrow \kappa$ such that $f(\alpha)$ is a cardinal, and let $\lambda=j(f)(\kappa)$. Take $\mathcal{U}$ and $\mathcal{U}^{\prime}$ as the $\lambda$ supercompactness measure and normal measure over $\kappa$ derived from $j$, i.e., $X \in \mathcal{U}$ iff $\langle j(\alpha) \mid \alpha<\lambda\rangle \in j(X)$, and $Y \in \mathcal{U}^{\prime}$ iff $\kappa \in j(Y)$. Then both $\mathcal{U}$ and $\mathcal{U}^{\prime}$ are elements of $M$. In addition, 
for $i: V \rightarrow \operatorname{Ult}(V, \mathcal{U})$ and $k: M \rightarrow \operatorname{Ult}(M, \mathcal{U})=M^{*}$, the closure properties of $M$ imply that $i(f)(\kappa)=k(f)(\kappa)$. Now, for $\ell: \operatorname{Ult}(V, \mathcal{U}) \rightarrow M$ the factor embedding, i.e., the elementary embedding such that $\ell \circ i=j$, we know that $\ell \mid \lambda=$ id. Also, by the definitions of $\ell$ and $\lambda, \ell(i(f)(\kappa))=j(f)(\kappa)=\lambda$. Further, if $i(f)(\kappa)<\lambda$, there must be some $\nu<\lambda$ (namely $i(f)(\kappa)$ ) such that $\ell(\nu)=\lambda$. However, since $\ell \uparrow \lambda=\mathrm{id}, \ell(\nu)=\nu$. This means that $i(f)(\kappa)=$ $\lambda=j(f)(\kappa)=k(f)(\kappa)$. And, because $\operatorname{cp}(j)=\kappa, M \vDash " f: \kappa \rightarrow \kappa$ and $j(f): j(\kappa) \rightarrow j(\kappa)$ are functions which agree below $\kappa "$. By elementarity, $M^{*} \vDash " k(f)$ and $k(j(f))$ agree below $k(\kappa)$ ", which immediately yields $k(f)(\kappa)=k(j(f))(\kappa)$. Putting all of the preceding together allows us to infer that $M \vDash " j(f): j(\kappa) \rightarrow j(\kappa), j(f)^{\prime \prime} \kappa \subseteq \kappa$, and there is a $\delta<j(\kappa)$ (namely $\kappa$ ) and an elementary embedding $k: M \rightarrow M^{*}$ with critical point $\kappa$ generated by a supercompact ultrafilter having rank below $j(\kappa)$ such that $\left(M^{*}\right)^{k(j(f))(\kappa)}=\left(M^{*}\right)^{k(f)(\kappa)} \subseteq M^{*}$ ". By reflection, $V \vDash$ "There is a $\delta<\kappa$ and an elementary embedding $k^{*}: V \rightarrow N$ with critical point $\delta$ generated by a supercompact ultrafilter having rank below $\kappa$ such that $f^{\prime \prime} \delta \subseteq \delta$ and $N^{k^{*}(f)(\delta)} \subseteq N$ ". Hence, $V \vDash$ " $\kappa$ is Woodin for supercompactness", so since $M^{\theta} \subseteq M, M \vDash$ " $\kappa$ is Woodin for supercompactness" as well. Consequently, $\kappa \in j(A)$, which means $A \in \mathcal{U}^{\prime}$. This completes the proof of Lemma 1.1.

Having completed the proof of Lemma 1.1, we now turn our attention to the proof of Theorem 1. We proceed in analogy to the proof of Theorem 5 given in [3], using the same definition for our forcing conditions as found there. Suppose $V \vDash$ "ZFC $+\kappa$ is Woodin for supercompactness". We define a reverse Easton iteration having length $\kappa$ which does nontrivial forcing only at those stages $\delta<\kappa$ which are measurable cardinals in $V$. If at such a $\delta$, some condition $p \in \mathbb{P}_{\delta}$ forces that $\delta$ is $<\gamma$ supercompact in $V^{\mathbb{P}_{\delta}}$ for $\gamma$ the next measurable cardinal above $\delta$ and the $<\gamma$ supercompactness of $\delta$ is indestructible under $\delta$-directed closed forcing having rank less than $\kappa$, then we stop the construction and use as our final model $\left(V_{\gamma}\right)^{V^{\mathbb{P}} \delta}$, assuming we have forced above $p$. Otherwise, we continue the iteration. In this case, there is some minimal $\alpha<\gamma$ such that the $\alpha$ supercompactness of $\delta$ is destroyed by some $\delta$-directed closed partial ordering $\mathbb{Q}$ of rank below $\kappa$. By the work of [3], we may assume that forcing with $\mathbb{Q}$ leaves no measurable cardinals in the half-open interval $(\alpha,|\mathbb{Q}|]$. We then let $\mathbb{P}_{\delta+1}=\mathbb{P}_{\delta} * \dot{\mathbb{Q}}$, where $\dot{\mathbb{Q}}$ is a term for such a $\mathbb{Q}$ of smallest possible rank.

By the same arguments as in [3], if there is a stage of forcing $\delta<\kappa$ at which we can stop the construction and use $\left(V_{\gamma}\right)^{V^{\mathbb{P}_{\delta}}}$ as our final model, then we have obtained a model for universal indestructibility for supercompactness containing a supercompact cardinal. Thus, it suffices to show that this is indeed what occurs. If this is not the case, then let $f: \kappa \rightarrow \kappa$ be defined inductively by $f(\delta)=0$ if $\delta$ is not a measurable cardinal, but for $\delta$ a mea- 
surable cardinal, $f(\delta)$ is the least inaccessible cardinal above $\max (\alpha, \beta, \gamma)$, where $V^{\mathbb{P}_{\delta}} \vDash " \gamma$ is the least measurable cardinal above $\delta$ and $\alpha$ is the smallest degree of supercompactness of $\delta$ below $\gamma$ that can be destroyed by some $\delta$-directed closed forcing $\mathbb{Q}$ which leaves no measurable cardinals in the halfopen interval $(\alpha,|\mathbb{Q}|]$ ", and $\beta$ is the smallest rank below $\kappa$ of such a $\mathbb{Q}$. By the fact $\kappa$ is Woodin for supercompactness, let $\delta<\kappa$ be such that $f^{\prime \prime} \delta \subseteq \delta$ and there is an elementary embedding $j: V \rightarrow M$ having critical point $\delta$ with $M^{j(f)(\delta)} \subseteq M$. Write $j\left(\mathbb{P}_{\delta}\right)=\mathbb{P}_{\delta} * \dot{\mathbb{Q}}_{\delta} * \dot{\mathbb{R}}$. By the definition of $f$, the closure properties of $M$, and the fact that $j(\kappa) \geq \kappa$ (in actuality, $j(\kappa)=\kappa$ ), we then see that in both $V^{\mathbb{P}_{\delta}}$ and $M^{\mathbb{P}_{\delta}}$, forcing with $\mathbb{Q}_{\delta}$ destroys the $\alpha$ supercompactness of $\delta$, where $\alpha$ is minimal below the least measurable cardinal above $\delta$ (which is the same in both $V^{\mathbb{P}_{\delta}}$ and $M^{\mathbb{P}_{\delta}}$ ), forcing with $\mathbb{Q}_{\delta}$ leaves no measurable cardinals in the half-open interval $\left(\alpha,\left|\mathbb{Q}_{\delta}\right|\right]$, and $\mathbb{Q}_{\delta}$ has smallest possible rank below $\kappa=j(\kappa)$. However, as in the proof of Theorem 5 of [3], the usual reverse Easton arguments show that if $G_{0}$ is $V$-generic over $\mathbb{P}_{\delta}$, $G_{1}$ is $V\left[G_{0}\right]$-generic over $\mathbb{Q}_{\delta}$, and $G_{2}$ is $V\left[G_{0}\right]\left[G_{1}\right]$-generic over $\mathbb{R}$, then $j$ lifts in $V\left[G_{0}\right]\left[G_{1}\right]\left[G_{2}\right]$ to $j: V\left[G_{0}\right] \rightarrow M\left[G_{0}\right]\left[G_{1}\right]\left[G_{2}\right]$. We may then find a master condition $q$ for $j^{\prime \prime} G_{1}$ in $V\left[G_{0}\right]\left[G_{1}\right]\left[G_{2}\right]$ with respect to the partial ordering $j\left(\mathbb{Q}_{\delta}\right)$, take $G_{3}$ as a $V\left[G_{0}\right]\left[G_{1}\right]\left[G_{2}\right]$-generic object containing $q$, and working in $V\left[G_{0}\right]\left[G_{1}\right]\left[G_{2}\right]\left[G_{3}\right]$, lift $j$ further to $j: V\left[G_{0}\right]\left[G_{1}\right] \rightarrow M\left[G_{0}\right]\left[G_{1}\right]\left[G_{2}\right]\left[G_{3}\right]$. As usual, $\mathcal{U}$ given by $X \in \mathcal{U}$ iff $\langle j(\beta) \mid \beta<\alpha\rangle \in j(X)$ is a supercompactness measure over $\left(P_{\delta}(\alpha)\right)^{V\left[G_{0}\right]\left[G_{1}\right]}$ which is present in $V\left[G_{0}\right]\left[G_{1}\right]\left[G_{2}\right]\left[G_{3}\right]$. However, by the closure properties of $\mathbb{R} * j\left(\dot{\mathbb{Q}}_{\delta}\right)$ in both $M\left[G_{0}\right]\left[G_{1}\right]$ and $V\left[G_{0}\right]\left[G_{1}\right]$, $\mathcal{U} \in V\left[G_{0}\right]\left[G_{1}\right]$. This contradicts that forcing with $\mathbb{Q}_{\delta}$ over $V^{\mathbb{P}_{\delta}}$ destroys the $\alpha$ supercompactness of $\delta$ and therefore completes the proof of Theorem 1 .

Theorems 7 and 8 from [3], in which models for universal indestructibility for strong compactness and universal indestructibility for strongness are constructed, also remain valid when forcing with the same partial orderings as in [3], using a cardinal Woodin for supercompactness and the method of proof given in Theorem 1 above. In addition, Theorem 6 of [3], where a model for universal indestructibility for supercompactness is constructed in which every Ramsey and weakly compact cardinal also satisfies the appropriate form of universal indestructibility, can be proven as well using a cardinal Woodin for supercompactness and the same partial ordering as in [3]. All of these models contain either a supercompact, strongly compact, or strong cardinal.

In order to prove Theorem 2, we need the following lemma, which is the analogue of Lemma 1.1 of [1]. It shows that the results of [8] are true for cardinals Woodin for supercompactness.

Lemma 1.2. Suppose $V \vDash " Z F C+\kappa$ is Woodin for supercompactness + $\mathbb{P}$ is a partial ordering such that $|\mathbb{P}|<\kappa$ ". Then $V^{\mathbb{P}} \vDash$ " $\kappa$ is Woodin for supercompactness". 
Proof. Suppose $p \in \mathbb{P}$ and $\dot{f}$ are such that $p \Vdash " \dot{f}: \kappa \rightarrow \kappa$ is a function with $\dot{f}(\alpha)$ a cardinal". Define in $V$ a function $g$ by $g(\alpha)=|\mathbb{P}|^{+}$if $\alpha \leq|\mathbb{P}|$, and $g(\alpha)=$ The least inaccessible cardinal above $\sup (\{\beta<\kappa \mid$ For some $q$ extending $p, q \Vdash$ " $\dot{f}(\alpha)=\beta "\})$ if $\alpha>|\mathbb{P}|$. Since $|\mathbb{P}|<\kappa$ and $\kappa$ is a regular limit of measurable cardinals, $g$ is a well-defined function whose values are always cardinals. It is then the case that $p \Vdash$ "For every $\alpha<\kappa, \dot{f}(\alpha)<g(\alpha)$ ". By the definitions of $g$ and Woodin for supercompactness, there is some $\delta<\kappa, \delta>|\mathbb{P}|$ and elementary embedding $j: V \rightarrow M$ having critical point $\delta$ such that $g^{\prime \prime} \delta \subseteq \delta$ and $M^{j(g)(\delta)} \subseteq M$. By the results of [8], since $|\mathbb{P}|<\delta$, $j$ lifts in $V^{\mathbb{P}}$ to $j: V^{\mathbb{P}} \rightarrow M^{j(\mathbb{P})}$. Then $p \Vdash$ "There is $\delta<\kappa$ and an elementary embedding $j: V^{\mathbb{P}} \rightarrow M^{j(\mathbb{P})}$ having critical point $\delta$ such that $\dot{f}^{\prime \prime} \delta \subseteq \delta$ and $\left(M^{j(\mathbb{P})}\right)^{j(\hat{f})(\delta)} \subseteq M^{j(\mathbb{P}) "}$. This completes the proof of Lemma 1.2.

We are just about ready to begin the proof of Theorem 2. Before doing so, however, we prove the following lemma.

LEMMA 1.3. If $\kappa$ is Woodin for supercompactness, then $\{\delta<\kappa \mid \delta$ is $<\kappa$ supercompact\} is unbounded in $\kappa$.

Proof. Assume towards a contradiction that $\{\delta<\kappa \mid \delta$ is $<\kappa$ supercompact $\}$ is bounded in $\kappa$. Let therefore $\alpha_{0}$ be such that for every $\alpha \geq \alpha_{0}, \theta_{\alpha}$ is the least cardinal below $\kappa$ with the property that $\alpha$ is not $\theta_{\alpha}$ supercompact. Define $f: \alpha \rightarrow \alpha$ by $f(\alpha)=\alpha_{0}^{+}$if $\alpha<\alpha_{0}$, and $f(\alpha)=$ The least inaccessible cardinal above $\theta_{\alpha}$ if $\alpha \geq \alpha_{0}$. By the fact $\kappa$ is Woodin for supercompactness, we may find $\delta<\kappa, \delta>\alpha_{0}^{+}$, and an elementary embedding $j: V \rightarrow M$ with critical point $\delta$ such that $f^{\prime \prime} \delta \subseteq \delta$ and $M^{j(f)(\delta)} \subseteq M$. By the closure properties of $M$ and the definition of $f$, it then immediately follows that $\delta$ is $\theta_{\delta}$ supercompact in both $V$ and $M$, a contradiction. This completes the proof of Lemma 1.3.

We are now ready to prove Theorem 2. Suppose once again that $V \vDash$ "ZFC $+\kappa$ is Woodin for supercompactness". By Lemma 1.3, let $\delta<\kappa$ be the smallest cardinal such that $V \vDash$ " $\delta$ is $<\kappa$ supercompact". Force with the partial ordering $\mathbb{P}$ of Theorem 1 of [2] defined with respect to $\delta$. By the results of [2], $V^{\mathbb{P}} \vDash$ " $\delta$ is the least measurable cardinal, $\delta$ is $<\kappa$ strongly compact, and the $<\kappa$ strong compactness of $\delta$ is indestructible under forcing with $\delta$-directed closed partial orderings having rank below $\kappa$ ". Since $\mathbb{P}$ may be defined so that $|\mathbb{P}|=\delta<\kappa$, by Lemma $1.2, V^{\mathbb{P}} \vDash " \kappa$ is Woodin for supercompactness". If we then let $\mathbb{Q}$ be the partial ordering of either Theorem 5 or Theorem 6 of [3] (both of which force universal indestructibility for supercompactness) or Theorem 7 of [3] (which forces universal indestructibility for strong compactness), with the first nontrivial stage of forcing taking place at or above the least weakly compact cardinal in $V^{\mathbb{P}}$ above $\delta$, then the arguments given in the proof of Theorem 1 of this paper show that the 
construction of $\mathbb{Q}$ terminates at some stage $\gamma<\kappa$. Let $\sigma<\kappa, \sigma>\gamma$, be the least weakly compact cardinal above $\gamma$ in $V^{\mathbb{P} * \dot{\mathbb{Q}}}$. By the same arguments as in [1], $\left(V_{\sigma}\right)^{V^{\mathbb{P} * \dot{\mathbb{Q}}}}$ is our model for either universal indestructibility for strong compactness or universal indestructibility for supercompactness (depending upon the exact definition of $\mathbb{Q}$ ) containing two strongly compact cardinals. This completes the proof of Theorem 2.

Since our constructions require implicit applications of Hamkins' Gap Forcing Theorem of [4] and [5], our proofs are going to require at the minimum as a hypothesis a supercompact limit of supercompact cardinals. Readers are urged to consult [3] for the explicit details. The exact consistency strength of universal indestructibility as discussed in this paper therefore remains unknown.

Added in proof (March 2007). It is possible to reduce the consistency strength of the assumptions used to establish Theorems 1 and 2 still further. Details can be found in our forthcoming paper "An Equiconsistency for Universal Indestructibility".

\section{References}

[1] A. Apter, Universal indestructibility is consistent with two strongly compact cardinals, Bull. Polish Acad. Sci. Math. 53 (2005), 131-135.

[2] A. Apter and M. Gitik, The least measurable can be strongly compact and indestructible, J. Symbolic Logic 63 (1998), 1404-1412.

[3] A. Apter and J. D. Hamkins, Universal indestructibility, Kobe J. Math. 16 (1999), $119-130$.

[4] J. D. Hamkins, Gap forcing, Israel J. Math. 125 (2001), 237-252.

[5] -, Gap forcing: generalizing the Lévy-Solovay theorem, Bull. Symbolic Logic 5 (1999), 264-272.

[6] A. Kanamori, The Higher Infinite, Springer, Berlin, 1994.

[7] R. Laver, Making the supercompactness of $\kappa$ indestructible under $\kappa$-directed closed forcing, Israel J. Math. 29 (1978), 385-388.

[8] A. Lévy and R. Solovay, Measurable cardinals and the continuum hypothesis, ibid. 5 (1967), 234-248.

Arthur W. Apter

Grigor Sargsyan

Department of Mathematics

Baruch College of CUNY

New York, NY 10010, U.S.A.

and

The CUNY Graduate Center, Mathematics

Group in Logic and the Methodology of Science

University of California

Berkeley, CA 94720, U.S.A.

E-mail: grigor@math.berkeley.edu http://math.berkeley.edu/ grigor

365 Fifth Avenue

New York, NY 10016, U.S.A.

E-mail: awapter@alum.mit.edu

http://faculty.baruch.cuny.edu/apter 